\title{
Skeletal Development During Childhood and Adolescence and the Effects of Physical Activity
}

\author{
Han C.G. Kemper
}

This paper reviews the growth and development of skeletal mass in youth and the effects of physical activity upon the bone mass in young people. The different methods to measure the bone mass are described such as anthropometrics, radiographics, dual energy $\mathrm{X}$-ray absorptiometry, quantitative computed tomography, and ultrasound. Two different mechanisms are important for the formation and plasticity of bone: a central hormonal mechanism (with estrogen production) and a local mechanism (based on mechanical forces of gravity and muscle contractions). This local mechanism is closely connected to physical activity patterns and therefore discussed in more detail. Thereafter the natural course of the development of the bone mass during youth is described, taking into account the pubertal stages of boys and girls and also the age at which the maximal bone mass (peak bone mineral density) will be reached. The last part is devoted to the effects of physical activity on bone mass based on results of randomized controlled trials. Although the number of experimental studies are scarce, significant effects of weight bearing activity and high impact strength training programs are shown on the side specific bone mineral density in both boys and girls.

Physical growth and development have been extensively investigated from prenatal growth to birth and from postnatal growth to adulthood by many longitudinal studies from all over the world. In 1955 Tanner (43) published the first edition of his book Growth at Adolescence and, in 1981, A History of the Study of Human Growth (44). Both books have been used as state of the art publications about human growth and development. The methods that are used in general to measure growth changes are mainly based on simple anthropometric measurements of the total body (body height, body mass) or of body segments (trunk height, limb lengths). Also breadth measurements (shoulder, hip, wrist, and knee), circumferences (head, trunk, hip, waist, and limbs), and skinfold measurements at different sites of the body are applied according to standard methods (47).

All of these measurements estimate different dimensions of the body but do not take into consideration changes in the composition of these body parts.

The author is with the Institute for Research in Extramural Medicine (EMGO), Faculty of Medicine at Vrije Universiteit, Van der Boechorststraat 7, 1081 BT Amsterdam, The Netherlands. 
Radiographic methods are used to indicate calcified cartilage and ossificated bone and to estimate skeletal maturation. Different methods have evolved to assess the rate of maturation or biological age from X-rays at wrist and knee. From a comparison of skeletal age with calendar age the child can be characterized as an early or late maturer (11).

In recent years, new methods have been developed to measure bone mass by energy absorption from gamma radiation by calcium in the bone. The methods mostly described in the literature are single photon absorptiometry (SPA), dual photon absorptiometry (DPA), dual energy X-ray absorptiometry (DEXA). and quantitative computed tomography (QCT).

Most people think that the skeleton is a passive structure; this is certainly not true. Bone is a vital, dynamic connective tissue, which adapts its structure to its function. To fulfil these structure-function relations adequately, bone is continuously being broken down and rebuilt in a process called remodeling. The mechanisms that underline bone formation are discussed in a separate paragraph.

Bone mass in general increases at the same rate during growth and development in boys and girls, but at the beginning of puberty, a sexual dimorphism occurs and bone mass increases faster in boys than in girls. Maximal bone mass is reached in the late teens and early 20s; thereafter, it gradually declines. This decrease is accelerated in women after menopause (Figure 1).

The average woman has a higher risk of osteoporosis than the average man for at least two reasons: First, women reach a lower maximal bone mass in their youth and, second, women lose bone at a higher rate after menopause.

Since not much is known about the natural development of bone mass during youth, the literature will be reviewed on (a) the changes in bone mass during prepubertal, circumpubertal, and postpubertal development; (b) the differences in bone

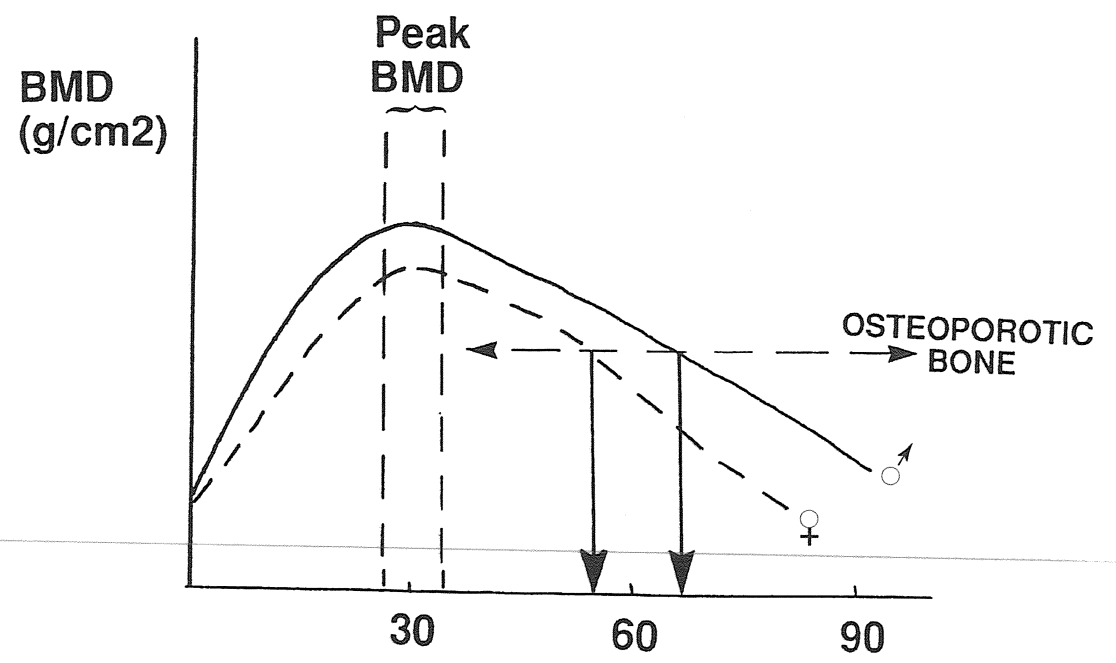

AGE (years)

Figure 1 - The development of bone mass in males and females with age; the osteoporotic fracture risk is usually reached at an earlier age in females than in males. 
development between boys and girls; and (c) the point of time at which the maximal amount of bone mass, or the so called peak bone mineral density (PBMD), is reached.

The final part of this article focuses on the effects of physical activity and the type of physical activity that is most effective with respect to bone adaptation. Most of the bone studies are aimed at prevention and retardation of bone loss in pre- and postmenopausal women. An important question remains whether it is possible to increase the bone mass during the growing years in boys and girls by physical activity in order to attain higher maximal bone mass at young adult age (41). Although there are only a few experimental studies the main results will be summarized.

\section{Methods of Measurements of Bone Mass}

\section{Anthropometrics}

Von Döbeln $(9,10)$ proposed a measure for estimation of the skeletal weight from height and four breadth measurements (left and right femur condyli and radio-ulnar width). This method is sound so long as it is used for estimating total weight of bone mass in comparison with muscle and fat mass estimated by skinfolds and circumferences in combination with height and weight. In the Netherlands, this concept is used to correct the body weight to body height relationship: The Dutch Heart Foundation constructed a reference scale (for ideal body weight) based on the Quetelet Index or Body Mass Index (QI or BMI: weight/height ${ }^{2}$ ) that included the possibility of calculating the ideal body weight taking the breadth of the femur condyle into consideration. However, this is a misuse of the skeletal component of this algorithm because an adjustment is made for the least variable of the three-component model of body composition, with lean and fat mass being the other components.

\section{Radiographics}

In November 1895, over 100 years ago, the German physicist Wilhelm Conrad Röntgen discovered gamma radiation and demonstrated a radiogram showing the bones of his own hand. He called this X radiation. The anatomist Albert von Kölliker connected Röntgen's name to this kind of radiation. Since then, X-rays have been widely used in medicine for detection of infectious diseases, pathologic neoplasmata, and traumatology.

A whole other field of applying radiographics is as a measure of biological age with respect to skeletal growth and development. Skeletal maturation begins as a process when rudiments of bones appear during embryonic life and is completed when skeletal form becomes comparatively stable in young adulthood. During maturation, there are increases in the types and numbers of specialized cells, including cartilage and fibrous tissue cells that form part of a bone (36). In 1950 Greulich and Pyle (18) published their radiographic atlas of skeletal development of the hand and wrist, with a second edition in 1959. Roche et al. (35) from his Longitudinal Study used the knee joint as bones of interest for determination of skeletal maturation (RocheWainer-Thissen method, RWT); however, most assessments of skeletal maturity are made from radiographs of the hand-wrist because this site has considerable advantages over other parts of the skeleton. These advantages stem from the little irradiation required, the ease of radiographic positioning, and the large number of bones included in the area. Therefore, the RWT method using the knee joint as a biological indicator for growth was extended with the hand-wrist method. In Europe, Tanner et 
al. (45), from the Institute of Child Health in London, published in 1975 their TannerWhitehouse II (TW2) method for the determination of growth also using X-ray photographs of the left hand, including 20 bones of the hand and wrist.

All these skeletal maturity scales are used to estimate the developmental or biological age of children, correcting for children who mature faster or slower than the average child with the same calendar age. In pediatrics, it can be used to predict adult height of children (mostly girls) whose parents expect them to be very tall and consider intervening in their growth by using hormones to close their endplates earlier.

\section{Dual Energy X-ray Absorptiometry (DEXA)}

Radiographs cannot easily quantify changes in bone density, because $30 \%$ of it has to be lost before it can be detected by X-ray. However, recent technical advances have made it possible to measure bone mass by energy absorption from gamma radiation in the bone. DEXA is now the most precise and widely used method of assessing bone density, as well as the preferred method, because scanning time is shorter than with dual photon absorptiometry (DPA). Also, resolution has been improved, and measurements can be made of the lumbar spine, femoral neck, and forearm, and for the total body.

From the DEXA method, two measures are calculated: the bone mineral content (BMC) and the bone mineral density (BMD). The BMC is the total amount of minerals in the selected bone in grams, and the BMD is the amount of grams of bone mineral divided by the area of the selected bone $\left(\mathrm{g} / \mathrm{cm}^{2}\right)$. The BMD however is not a real measure of bone density $\left(\mathrm{g} / \mathrm{cm}^{3}\right)$. This is therefore called area density.

In growth, bones not only increase their area but also their volume. These size changes influence the areal BMD. Therefore, attempts have been made to estimate the volume of the bone of interest and to correct for this bone size effect by an additional measure of bone mineral apparent density (BMAD; ref. 38).

\section{Quantitative Computed Tomography (QCT)}

QCT systems have been adapted for estimation of bone mineral content allowing cortical bone to be separated from trabecular bone. Furthermore, it provides us with a true measure of total, cortical, or trabecular bone mineral volumetric density $(\mathrm{mg} \times$ $\mathrm{mm}^{3}$ ). However, the equipment is more expensive and exposes patients to high radiation doses. A peripheral QCT system is now available for the forearm with a lower dose of radiation. Recently, Frost (12) showed that the breaking strength of rat femurs is more highly correlated with QCT derived bone strength index $(r=0.94)$ than with $\operatorname{BMD}(r=0.80)$.

\section{Ultrasound}

Ultrasound measurements have been available since 1984 for the calcaneus and have the potential for wide-spread clinical applications.

\section{Mechanisms of Bone Formation}

Movement is the result of electric impulses being passed from the central nervous system to the skeletal muscles. These muscles contract (shorten) in order to move 
body parts with respect to each other (arms, legs, head, and trunk) and/or the whole body with respect to the surroundings (walking, cycling, swimming). Exercise is not necessarily dynamic - sometimes muscles contract without causing movements but increase their tension as in static exercises such as standing, active sitting, or pushing
against a wall.

Both the duration and intensity of exercise play a role in the physical load placed on the body. Low-intensity, long-lasting exercise increases ventilation and circulation to meet oxygen demand for delivery energy to the active muscles. This is important for a better capillarization and oxygen delivery to the muscle. Highintensity, short-lasting exercise is important for the development of muscle and bone mass. Results show that of these two factors, it is not the duration of exercise which is the key factor affecting bone health but the intensity of the forces that act upon the bones. Weight-bearing activities, such as walking, running, and dancing, have more effect on bone health of the legs and vertebrae of the lower back than have swimming and bicycling, although all activities need approximately the same amount of energy when performed for identical lengths of time. This difference in effect on bone health is in contrast to the effects of these activities on the lungs, heart, and circulation: If performed with the same intensity and duration, swimming has the same effect as running on the oxygen transport system.

Two different mechanisms seem to act on bone mass: central hormonal factors, such as estrogen production, and local mechanical factors, such as the muscle forces exerted on the bones of the skeleton during contraction and the forces of gravity that act on the entire body during standing and other weight-bearing activities (40):

a. Central hormonal factors maintain serum calcium concentrations within a limited range. Calcium is one of the most common ions in the human body, and almost $99 \%$ of body calcium is deposited in the skeleton. Estrogens suppress the activity of osteoclasts, the bone-resorbing cells, and thus help to maintain bone mass. During exercise, serum concentrations of testosterone, and estrogen are elevated, influencing calcium homeostasis and the activity of osteoclasts and osteoblasts. Hormonal replacement therapy in women after the menopause makes use of this action of
estrogen.

b. The local mechanical forces of exercise cause (1) stress on the bone and calcium accumulation on the concave side of the bending bone, and (2) microtraumata that are removed by osteoclasts and repaired by osteoblasts.

The supposed mechanisms behind the local mechanical forces are the following:

- During flexion the bone acts like a piezo-electric crystal while accumulating calcium at the concave (i.e., negative loaded) side;

- Mechanical demands, occurring by overload, are sensed in the bone by osteocytes via strain-derived flows of interstitial fluid. They stimulate the osteoclasts in removing the damaged structures and at the same time the osteoblasts repair the structure of the bone matrix (7). In the case of a too strong or too often damaged bone, the process of repairing falls behind the process of removal, and microfracture will occur. When the mechanical load falls below the fracture intensity, remodeling activities are stimulated and result in bone hypertropy. Remodeling of the bone after a change in mechanical load by weight bearing activities (including experiments with added extra weights) has been proved in experimental studies in a great number of animals (26). Moreover, in some of these experiments, it has been shown that the effects are proportional to the 
intensity of the (extra) load. The amount of hypertrophy seems also to depend on the difference between the extra load and the load to the bone before the extra load was added.

- Not much is known about the interaction between central-hormonal and local-mechanical factors. However, physical activity leads to an increase of serum estrogen levels; this diminishes the sensitivity of the bone for the parathyroid hormone and the activity of the osteoclasts; when bone mass thus increases, more calcium $\left(\mathrm{Ca}^{2+}\right)$ and phosphorus $(\mathrm{P})$ are resorbed from the blood. This lowering of $\mathrm{Ca}^{2+}$ and $\mathrm{P}$ concentrations in the blood then stimulates the parathyroid hormone; the latter inhibits vitamin D production, stimulates calcium absorption, and decreases calcium secretion.

As long as the forces exerted on the bones remain weaker than those needed to cause a macro fracture (referred to as the fracture limit), this remodeling process is able to adapt the bone to the external biomechanical stress and bring about bone thickening (hypertrophy). During long periods of inactivity, such as prolonged bed rest, the bone becomes atrophic as a result of relatively higher osteoclast activity compared to osteoblast activity. The central hormonal system and the local mechanical system interact to optimize the function of the skeletal system. In the case of exercise, mechanical factors seem to be most important for affecting bone mass.

Animal experiments (37) in an ulna-model of roosters have shown that loading of bone a few times (four times) a day can prevent bone loss, and that a frequency of loading ( 36 per day) results in an optimal increase in bone mass. Bone mass is not further increased by increasing the daily frequency of bone loading to 360 or even 1,800 times per day (Figure 2). Therefore in humans, short bursts of

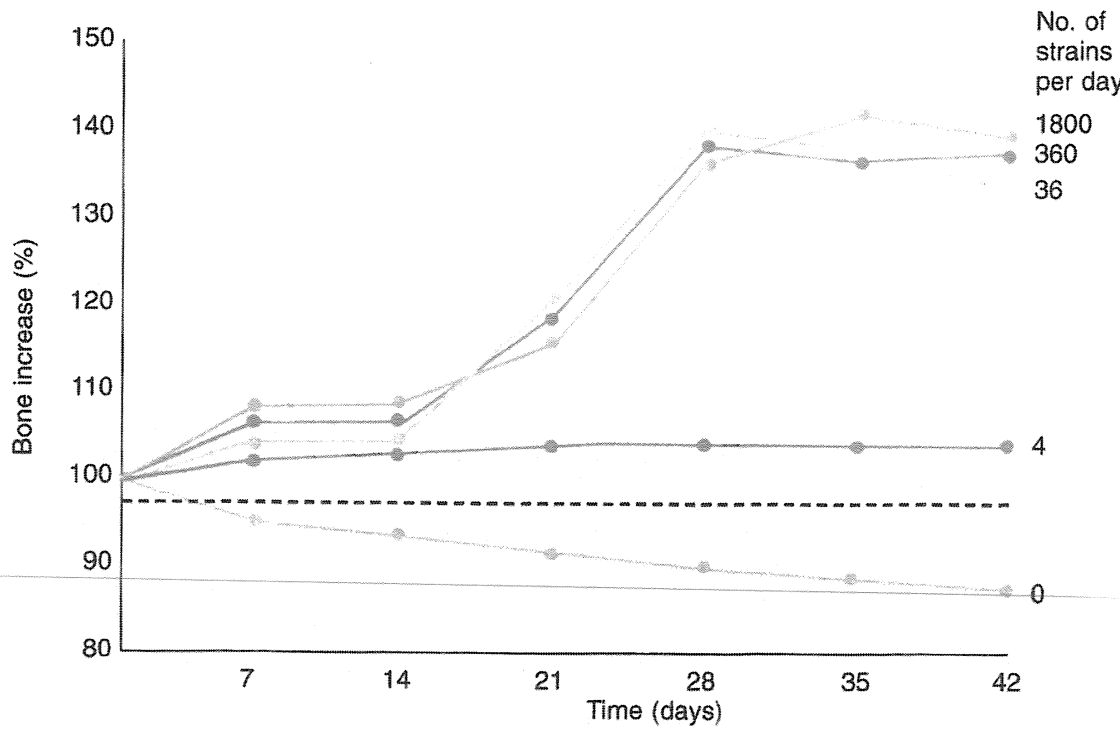

Figure 2 - Experiments in animals show that loading bones at a frequency of four times a day over a period of 42 days is sufficient to maintain bone mass and that an optimal rate is 36 times a day, which increases bone mass by $\mathbf{4 0 \%}$. A further increase in frequency to 360 or 1,800 times a day did not have more effect(after Rubin and Lanyon, ref. 37). 
explosive exercise, such as skipping, stair climbing, and jumping, are supposedly more effective for bone development than popular forms of exercise such as walking, jogging, bicycling, and swimming.

Bone, therefore, appears to react best to exercise that is characterized by a pattern of unexpected and irregular high loads with a relatively low frequency and short duration. This is quite different from endurance exercise designed to improve aerobic function, which needs a load of long duration (or high frequency) and low intensity. For comparison, Figure 3 shows an example of a typical and effective exercise for loading bone (skipping) and an effective exercise for loading the oxygen transport system (jogging). Extrapolated from the results of animal studies, skipping for 1 min a day ( ing bone mass, whereas jogging for $1 \mathrm{hr}$ a day (two times $30 \mathrm{~min}$ ) is more effective for the development of the oxygen transport system. Exercise that is effective in maintaining bone mass seems to take a lot less time than endurance exercise!

\section{Natural Course of Bone Mass Development}

Although in Figure 1 the general course of bone mass was outlined, not much is known about the exact timing of the age at which the maximal amount of bone mass is reached. Therefore, I first review the literature about bone development in boys and girls before puberty. Second, I estimate the importance of the pubertal period in the total development of bone mass. Third, I answer the question about the age at which maximal or peak bone mineral density occurs in males and females.

\section{Development of Bone Density Before Puberty}

Six cross-sectional studies $(5,13,15,17,42)$ and a 1-year longitudinal study (46) conclude that between boys and girls, there is no significant difference between
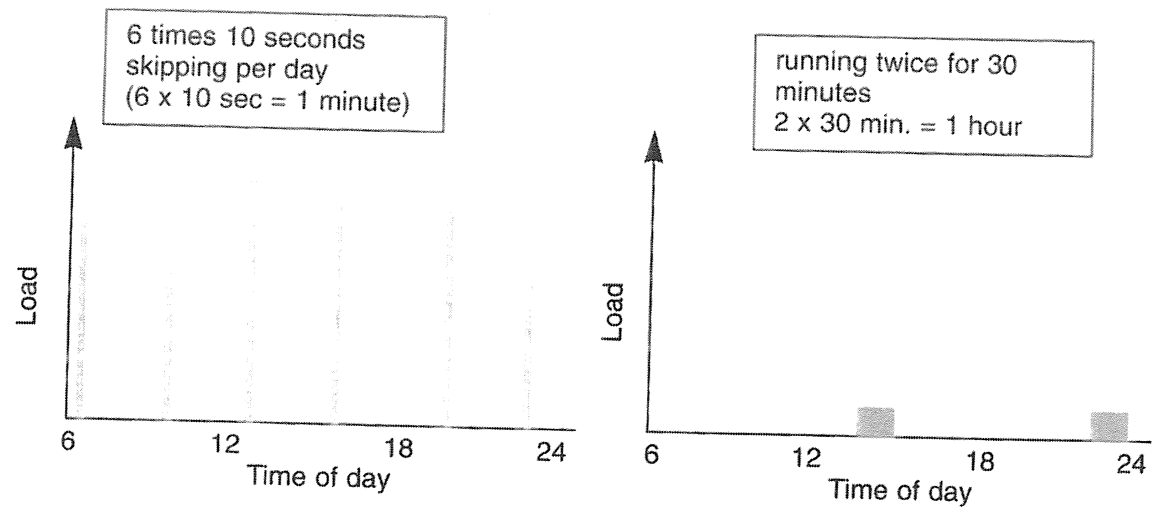

Figure 3-Comparison of two types of exercise with different effects on the musculoskeletal and the cardio-respiratory system. Short explosive exercise (A), such as skipping six times a day for $10 \mathrm{~s}$ (total exercise time per day is $60 \mathrm{~s}$ ), is effective for bone and muscle strength, whereas low-intensity exercise $(B)$ of long duration, such as jog-

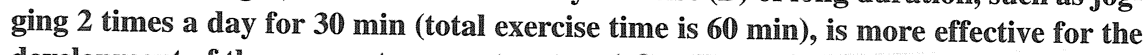
development of the oxygen transport system (after Kemper, ref. 23). 
the bone mineral density of the radius and the lumbar spine. This indicates that the development of BMD before puberty is not dependent on steroids.

Although there is a trend for a gradual increase from birth to puberty in bone mass, from the seven reviewed publications it is not possible to make a quantitative estimation of the proportional contribution of this time window to the total (adult) bone mass. Although before puberty, there is no difference in BMD between boys and girls, there is a sex difference apparent with a greater bone mass accumulation rate from 11-14 years in girls and from 13-17 years in boys (46).

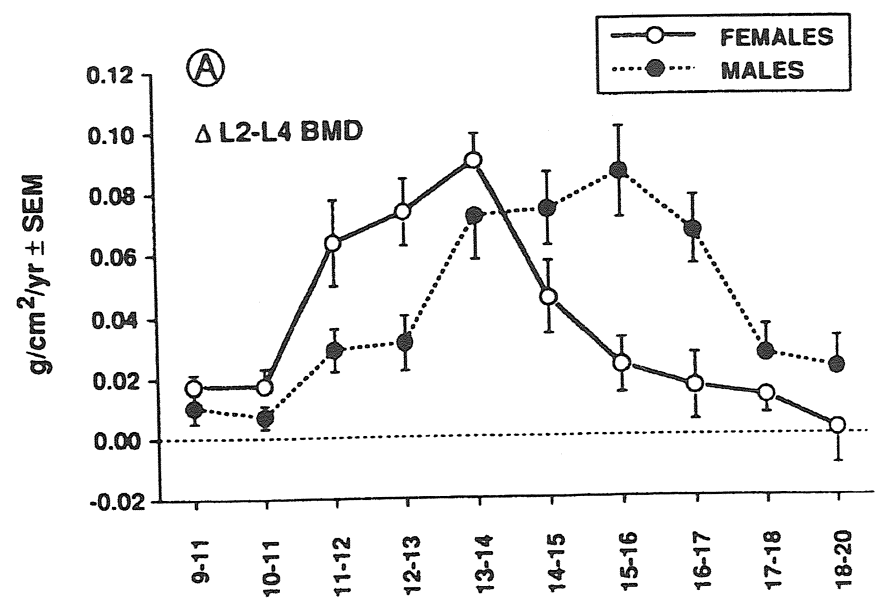

AGE RANGE

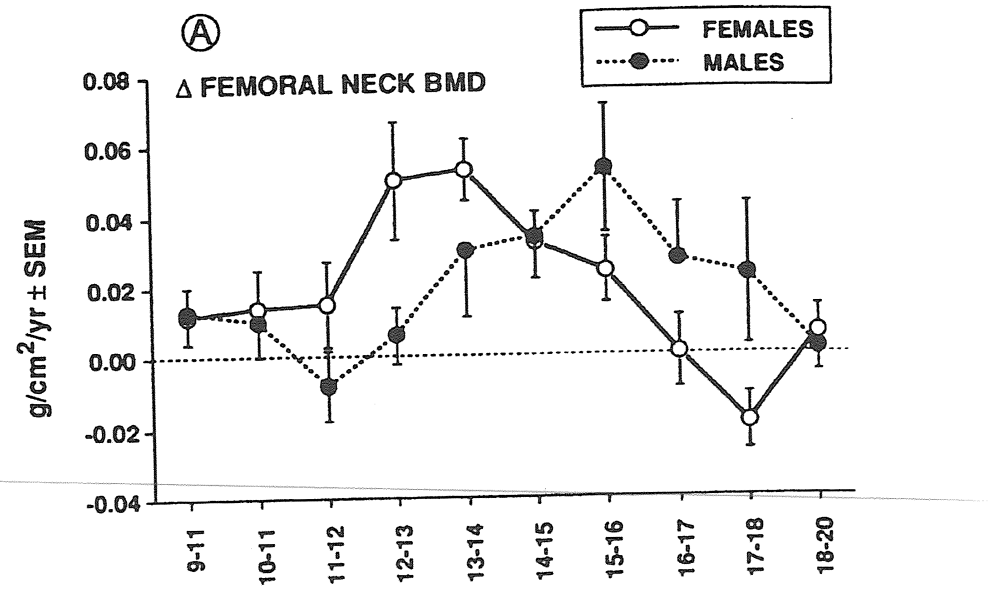

AGE RANGE

Figure 4 - Bone mass gain (g/ $\mathrm{cm}^{2} /$ year) in $\mathrm{BMD}$ of lumbar region and femoral neck in boys and girls during adolescence (after Theintz et al., ref. 46). 


\section{Development of Bone Density During Puberty}

Puberty is a relative short period of 3-5 years in the life of boys and girls. This short period seems to be a very important one for the development of bone mass if we review the literature. The results of six cross-sectional studies $(5,13,14,15,17$, 19) are shown in Figure 5. The increase of lumbar BMD in girls varies between 17 and $70 \%$ and in boys between 38 and $75 \%$ of total adult values. Riis et al. (34) found an increase of $31 \%$ in the radial region during the puberty of boys.

The high variation in the results can be attributed to several factors: (a) differences in the classification of puberty, (b) confounding factors such as nutritional and/or activity patterns that are different for the populations studied, and (c)

the possible influence of early or late maturation: Early maturation coincides with a relatively longer exposition to sex specific hormones than late maturation; estrogen levels in girls and testosterone levels in boys seem to be related to bone mass
development.

These cross-sectional data shown in Figure 5 suggest that in boys and girls, the pubertal years add sometimes $30-75 \%$ to total bone.

However, Bailey et al. (2) reported that the BMD changes should be interpreted with caution because of the methods used. Determination of BMD by projectional methods like Dual X-ray Absorptiometry (DEXA) provide areal densities $\left(\mathrm{g} / \mathrm{cm}^{2}\right)$ that are confounded by the earlier mentioned size changes accompanying growth. Consequently, calculated volumetric BMD percentage increases are substantially less than the corresponding area BMD value increases.

This dimensional consideration explains why Gilsanz et al. (14) showed the lowest increase $(17 \%)$, since they were the only ones that used the quantified computerized tomography (QCT) method to measure BMD, and this method provides
real volumetric BMDs.

The BMD changes during the growth period that are reported in the literature, which indicates that around puberty $50 \%$ of BMD is accreted, are measured with DEXA and must therefore be doubted. The only study with the QCT method reports a $15 \%$ volume $\mathrm{BMD}$ increase in pubertal girls, which seems to be a more
realistic value.

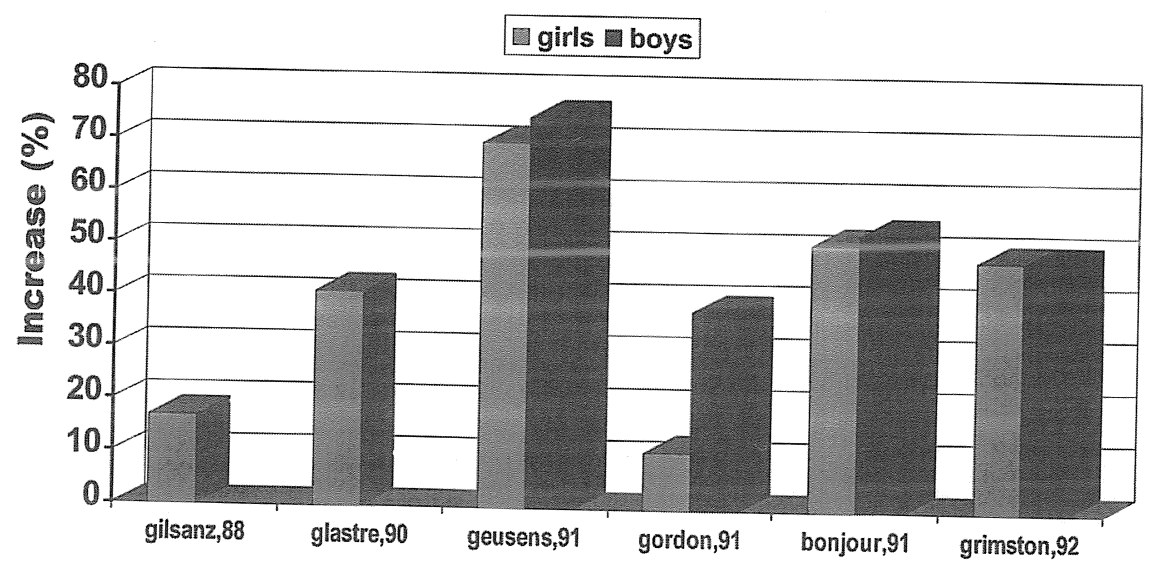

Figure 5 - Estimation of percentage increase in lumbar bone mineral density reported in six cross-sectional studies in boys and girls during their pubertal years. 


\section{Maximal Bone Mass (Peak Bone Mineral Density, PBMD)}

Most of the anatomical structures and physiological functions, such as muscle mass, cardio-respiratory functions, immune system, and central nervous system, show a typical pattern over time. This is characterized by a steep increase during the growth period until the age of 20 years and, thereafter, a much slower decrease and gradual decline during aging (24). This pattern implies that there is a point or period in time when the human functions reach their maximal capacities. The question is whether there is a similar pattern observable in the development of bone mass, and if so, at what point in time of life peak bone mineral density (PBMD) occurs.

From 12 cross-sectional studies that have been published since 1981, 7 have been performed with girls and 5 with both boys and girls.

In principle a cross-sectional design is not adequate to indicate individual changes over time. It also has methodological constraints (such as cohort effects, secular trends, etc.). With these flaws in mind, the results of six cross-sectional studies, with acceptable methodology and with sufficient information from the publications, will be considered $(5,6,13-15,32)$.

They report an age period of reaching PBMD in girls between 16 and 23 and, in boys, between 16 and 25 years. In Table 1, the results of estimated age of peak bone mineral density (PBMD) of each of the six valid studies are given separately for boys and girls.

Eight longitudinal studies investigated the development of BMD and PBMD. All of them used female subjects. From a methodological point of view the quality of three studies can be questioned seriously. These studies tend to confirm the cross-sectional results that PBMD occurs before the age of 20. However the two high quality studies from Davies et al. (8) with a follow up of 4 years, and from Recker et al.(31) with a follow up of 5 years, show very clearly that at least in females, the age of PBMD is reached much later than 20 years: Lumbar, radial, and total BMD reach the highest values around the age of 30 years.

In Table 2, the estimated age at PBMD of three low quality $(29,33,39)$ and two high quality $(8,31)$ longitudinal studies is summarized.

Because no data are available for males, it remains unknown at what age PBMD is reached in males.

Table 1 Cross-Sectional Studies-Twelve Studies Between 1981 and 1992

Age at PBMD (years)

Study

Gilanz et al. (14)

Buchanan et al. (6)

Glastre et al. (15)

Geusens et al. (13)

Bonjour et al. (5)

Rico et al. (32)

Females Males

$\begin{array}{cc}16-17 & - \\ 15-23 & - \\ >15 & >15 \\ 16-20 & 21-25 \\ 14-15 & 17-18 \\ 15-19 & -\end{array}$


Table 2 Longitudinal Studies-Eight Studies of Females

Three studies that are invalid

Age at PBMD (years)

Riggs and Melton (33)

Moen et al. (29)

17-18

Slemenda et al. (34)

$<20$

Two studies that are invalid

Age at PBMD (years)

Davies et al. (8)

$>26$

Recker et al. (31)

The discrepancies between the results of cross-sectional and longitudinal studies should be attributed to confounding factors. In general high quality crosssectional studies tend to establish PBMD in females between 16 and 25 years of age and the high quality longitudinal investigations, much later, around the age of 30. Because longitudinal data are more valid to detect age changes, it is more likely that PBMD in females is occurring not in their late teens but in their late 20 s.

Recently Bailey (1) investigated bone mineral accretion in growing children from the Saskatchewan Pediatric Bone Mineral Accrual Study. To investigate how bone mineral at clinically important sites proceeds in relation to maturation, distance and velocity curves for body height and bone mineral content (BMC) were made in both boys and girls, measured every 6 months during 6 years.

Figure 6 shows the results: In both boys and girls, over $35 \%$ of total body $\mathrm{BMC}$ was laid down during the 4-year circumpubertal period, and peak BMC was reached about 1-1.5 year after peak height velocity (PHV).

\section{Effects of Physical Activity and Physical Fitness on Bone Mass}

Longitudinal studies that include interventions with extra physical activity are indispensable in proving that bone mass can be influenced by the daily activity pattern of the subjects involved. The majority of these intervention studies are done in females older than 45 years in order to investigate if exercise protocols can prevent postmenarchal bone loss and later osteoporosis. The preferred methodology is the so called randomized controlled trials (RCT).

In a recent meta-analysis of RCTs, the effects of exercise training programs in pre- and postmenopausal women on BMD of the lumbar spine (LS) and the femoral neck $(\mathrm{FN})$ were summarized (49). The study treatment effect was defined as the difference between percentage change in BMD per year in the training and the control group. Seventeen articles were included. The summary treatment effects were in premenopausal women $0.9 \%$ (95\% CI: $0.4-1.4)$ in LS and $0.9 \%$ $(0.3-1.5)$ and, in postmenopausal women, $0.9(0.4-1.3)$ in LS and $1.0(0.4-1.5)$ in 


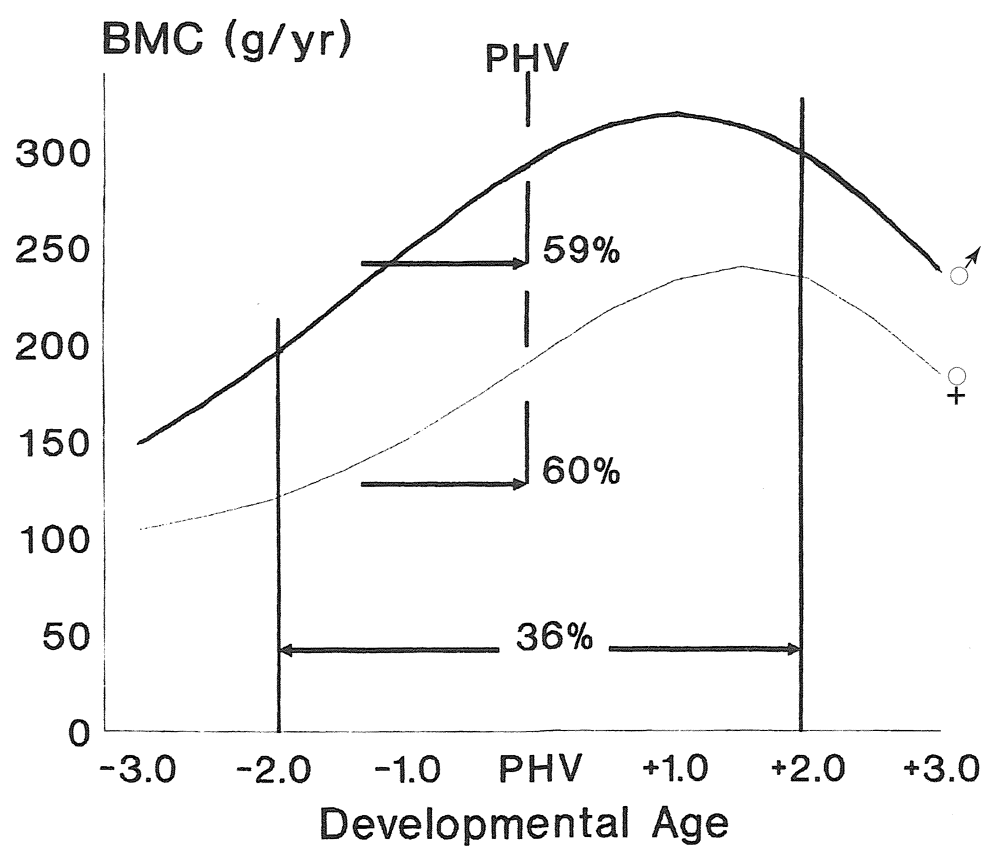

Figure 6 - Total body bone mineral content velocity curves of boys and girls aligned on age at PHV (after Bailey, ref. 1).

FN. It showed that exercise prevented almost $1 \%$ BMD loss per year in both preand postmenopausal women.

The number of RCTs in young subjects however are scarce: Three studies on girls $(4,16,30)$ and two on boys are valid for review $(3,28)$.

The boys' study of Margulies (28)—with 268 military recruits, age 18-21 involved in intensive training $8 \mathrm{hr}$ per day per week - had no control group, and the period of follow-up was relatively short (14 weeks). More importantly, about $40 \%$ of the subjects could not comply because of stress fractures.

In 1998, Bradney et al. (3) published a study in prepubertal boys comparing an 8 months, three times per week 30 -min program consisting of weight bearing exercise with a control group matched for age, height, weight, and BMD.

The increase in BMD was site specific and twice that in controls in lumbar spine, legs, and total body (Figure 7).

Gleeson et al. (16) performed a 1-year three times per week weight training program of 30 min duration, with an intensity of $60 \%$ of the one repetition maximum in 34 women (24-46 years). They compared the bone density in lumbar spine and calcaneus with 38 controls. No changes in BMD could be found in either group. Blimkie et al.(4) found also nonsignificant changes in younger girls (14-18 years) following a weight training program over a shorter period of 26 weeks.

A 10-month intervention in premenarchal girls by Morris et al. (30), with high impact strength-building exercise, showed a significant increase at all four bone sites of interest (proximal femur, neck of femur, lumbar spine, and total body). This increase was accompanied by a decrease in fat mass and a gain in lean mass, shoulder, knee, and grip strength (Figure 8). 
Changes in Areal Bone Mineral Density

(\% per month)

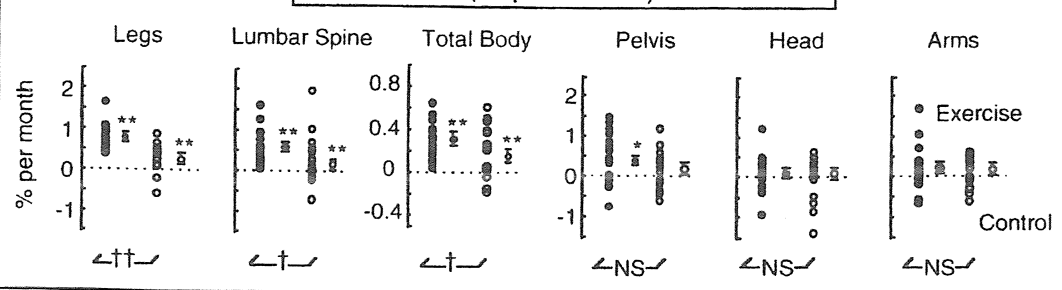

Figure 7 - Changes in areal $B M D$ as a percentage per month in six regions in the exercise and control group. $* \mathrm{p}<.05$; $* * \mathrm{p}<.01$, change from baseline; $+\mathrm{p}<.05$; $++\mathrm{p}<$ .01 (after Bradney et allo, ref. 3).
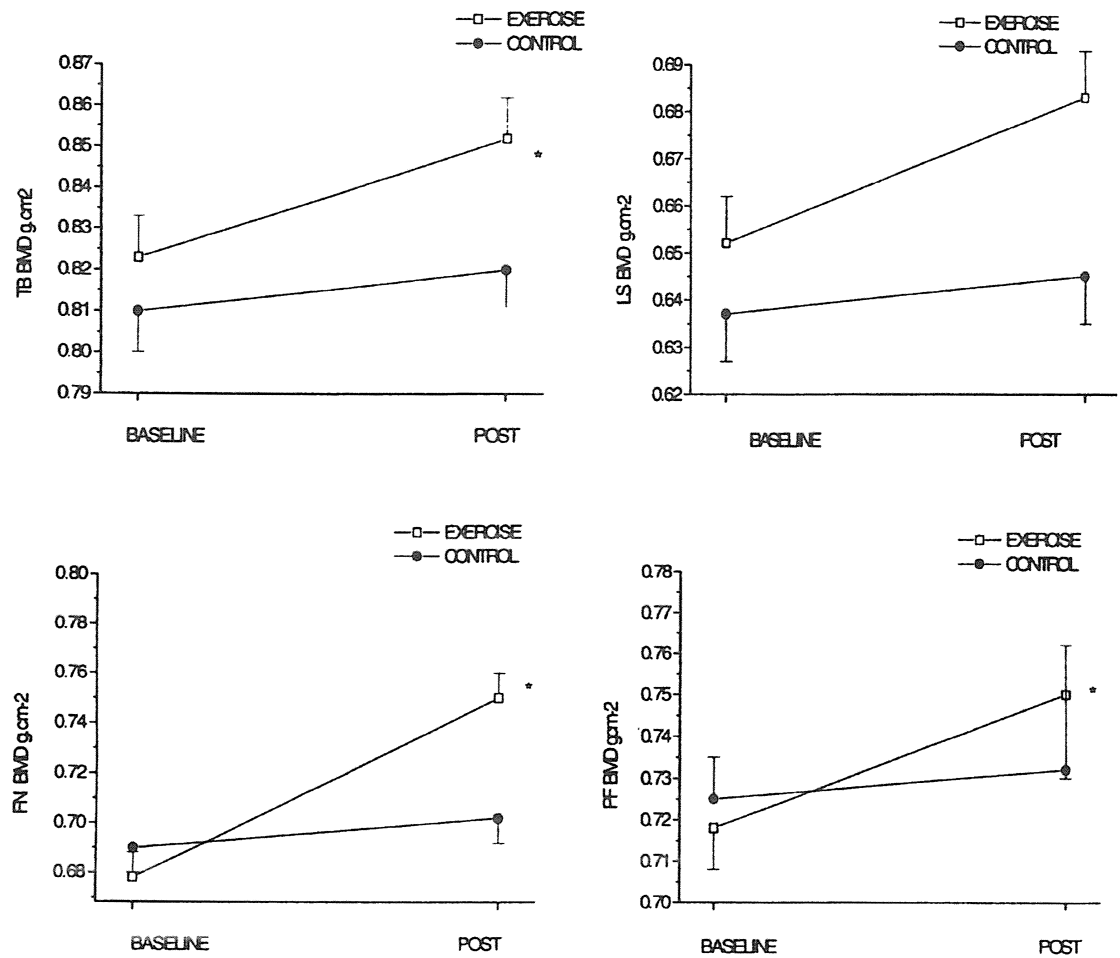

Figure 8 - Absolute change in BMD across 10 months in exercise and control group; TB = total body; LS-lumbar spine; $\mathrm{FN}=$ femoral neck and $\mathrm{PF}=$ proximal femur; ${ }^{*} p<$ .05 (after Morris et al., ref. 30).

Non-experimental results are available from the Amsterdam Growth and Health Longitudinal Study (48). About 200 males and females were measured longitudinally from age 13 to 27 . In this study, measurements were taken of habitual physical activity and nutritional intake on six occasions. At age 27 the BMD of the lumbar region was measured by DEXA. 
The longitudinal information of weight bearing activity and calcium intake were considered over three periods: the adolescent period from 13-18 years, the period between 13 and 22 years, and the total period between the age of 13 and 27 years.

Results of multiple regression analysis showed that in both sexes, weight bearing activity and body mass were significant positive contributors to the prediction of BMD at age 27. Calcium intake never appeared to be a significant predictor of BMD in the three periods. From these results, it can be concluded that BMD in the lumbar spine at age 27 may be influenced by body mass and a high level of weight bearing physical activity carried out during youth.

To address the question about what the most important factor is for bone mass development during youth in the same study, the physical activity data were scored in two different ways: (a) by calculating the total weekly energy expenditure of all weight bearing activities (expressed as the number of weight bearing METs/week), and (b) by calculating a score that takes into account the ground reaction forces at weight bearing activities as multiples of body weight, irrespective of the frequency and the duration of the activity (i.e., giving a weighted peak strain score).

The two different habitual physical activity scores were again calculated for each subject over three time periods: the adolescent period (four annual measurements between 13 and 17 years of age), the young adult period (two measurements between 17 and 22 years of age), and the adult period (two measurements between 22 and 27 years of age).

Linear regression analyses were performed to analyze the relation between BMD at age 28 and the physical activity scores over three foregoing periods. The physical activity scores were entered in the regression model as independent variables, and gender was added to the model as a covariate. In Figure 9, the standard

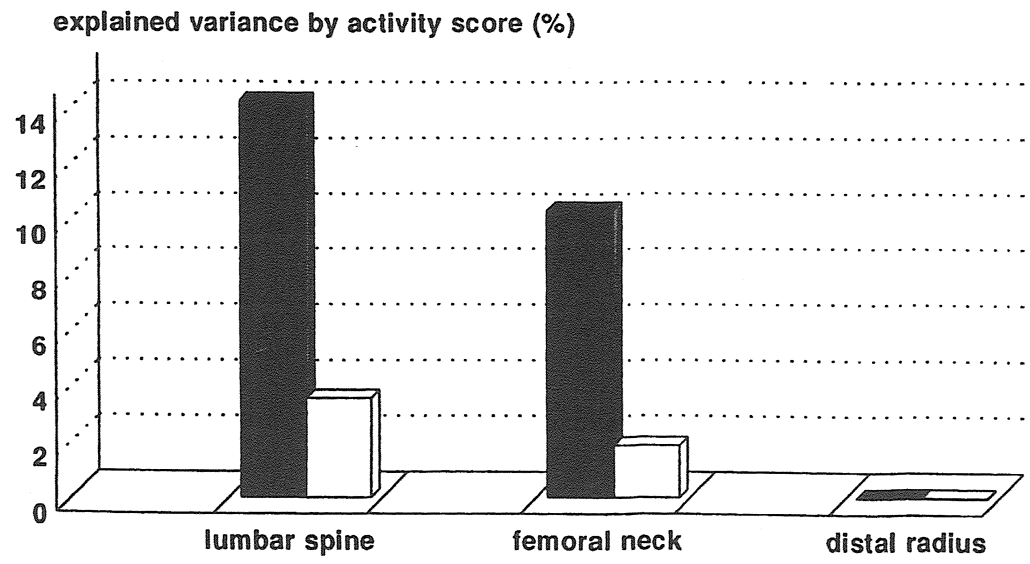

Peak-score $\square$ Met-score

Figure 9 - The relationship of BMD in the lumbar spine at age 27 years with metabolic physical activity (MET) score and mechanic physical activity (Peak) score during three different preceding periods in $\mathbf{1 8 2}$ males and females from the Amsterdam Growth and Health Study (22). 
regression coefficients of lumbar BMD and femoral neck, BMD, respectively, are given for the MET score and the peak strain scores, and for the three different periods.

The results show that the time period over which the physical activity scores were taken came closer to the BMD measurement at age 27 , and the more important became the peak strain score of physical activity. For this mechanical component of physical activity, the explained variance of BMD increased from $2 \%$ during adolescence to $13 \%$ in adulthood. For the metabolic score of physical activity the explained variance decreased from $6 \%$ during adolescence to $1 \%$ in adulthood for both sexes (20). This strongly supports the validity of the results of animal studies in human subjects.

The preventive effect of peak strain, however, has to be confirmed in youth in a true experimental design, since the significant differences in BMD can still be explained by self-selection of activity levels during the growing years.

Recent results from Mirwald et al. (27) presented at the first international conference on Children's Bone Health in Maastricht comparing active subjects (top quartile) with inactive subjects (bottom quartile) suggest that a modifiable lifestyle factor like physical activity plays a role in the optimization of bone mineral acquisition at the lumbar spine in boys and girls during the adolescent growth spurt. A cross-sectional study in female tennis and squash players (21) showed that training started in puberty is maximally beneficial for mineralization of the bone of the playing arm. This training effect on BMD remained into adulthood (age 21-30 years) after 4 years of cessation of the training (25).

\section{Conclusions}

Bone mass increases rapidly during growth and development. The mechanism seems to be dependent on centrally regulated hormonal factors, locally determined mechanical factors, and an interaction between the hormonal and mechanical factors.

The quantitative increase of BMD during growth, measured by energy absorption methods such as DPA, SPA and DEXA, gives an overestimation, because these measures do not take into consideration differences in dimensional growth of the bones in question.

Before the age of puberty (around 12 in girls and 13 in boys), no significant differences in BMD between boys and girls are demonstrated. Girls show greater bone mass accumulation rate at an earlier age (11-14 years) than boys (13-17 years).

During the pubertal growth spurt, it is now clear that the increase in BMC on the average is $35 \%$ of total lifetime BMC increase. The clinical significance of this high percentage is that as much BMC is laid down during the four adolescent growing years as most people loose during their adult life.

Investigations that measured BMD longitudinally indicate that boys and girls reach their peak BMD in their late 20 s and not in their late teens. In both sexes the greatest change in BMC per year occurs 1 or 2 years after PHV.

There are at least two exercise-related strategies to prevent osteoporosis (Figure 10). One preventive strategy is to increase bone accrual during youth by increasing the amount of exercise in order to achieve a greater peak bone mass. A second strategy is to ensure that adults maintain a physically active lifestyle until old age, thus minimizing bone loss during aging. In this way, exercise delays the age at which the osteoporotic fracture limit is reached. 


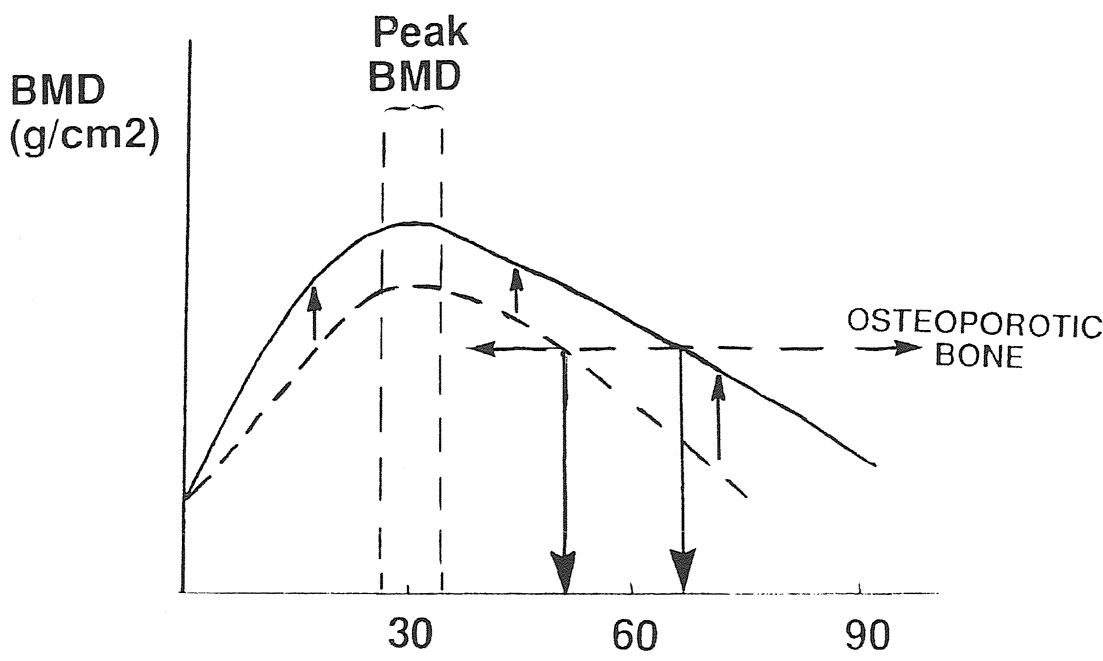

AGE (years)

Figure 10 - The possible effects of lifetime exercise on the developmental curve of $\mathrm{BMD}$ : the average curve of inactive people (interrupted line) is shifted to the top-right (solid line) resulting in a higher $\mathrm{BMD}$ at any age and crossing the osteoporotic limit at a later age.

In young males and females the effects of exercise intervention on BMD are scarce. Recent experimental studies show significant effects of weight bearing activity and high impact strength training programs on the side specific BMD in both boys and girls. The earlier a child starts with physical activity, the more bone is accumulated.

A preventive effect of weight bearing activities on the PBMD is also shown in the Amsterdam Growth and Health Longitudinal study: Both 27-year-old males and females, with relative high levels of peak strain weight-bearing physical activity pattern during the foregoing 15 years, show significantly higher PBMD in their lumbar spine than their inactive counterparts.

Further research is required to establish the most effective type of exercise for increasing bone mass and true experimental studies that are aimed at the possibility of increasing PBMD in both sexes in order to attain optimal maximal bone mass at young adult age.

\section{References}

1. Bailey, D.A. The Saskatchewan Pediatric Bone Mineral Accrual Study: bone mineral acquisition during the growing years. Int. J. Sports Med. 18:S191-S195, 1997.

2. Bailey, D.A., D. Drinkwater, R. Faulkner, and H. McKay. Proximal femur bone mineral changes in growing children: dimensional considerations. Pediatr. Exerc. Sci. 5:388, 1993.

3. Bradney, M., G. Pearce, G. Naughton, C. Sullivan, S. Bass, T. Beck, J. Carlson, and E. Seeman. Moderate exercise during growth in prepubertal boys: changes in bone mass, 
size, volumetric density and bone strength: a controlled prospective study. IBMR. 12:1814-1821, 1998.

4. Blimkie, C.J., S. Rice, J. Webber, J. Martin, D. Levy, and D. Parker. Bone density, physical activity, fitness, anthropometry, gynaecologic, endocrine and nutrition status in adolescent girls. In: Pediatric Work Physiology, J. Coudert and E. van Praagh (Eds.). Paris: Masson, 1993, pp. 201-204.

5. Bonjour, J.F., G. Theintz, B. Buchs, D. Slosman, and R. Rizzoli. Critical years and stages of puberty for spinal and femoral bone mass accumulation during adolescence. J. Clin. Endocrin. Soc. 73:555-563, 1991.

6. Buchanan, J.R., C. Meyers, T. Lloyd, and R.B. Greer III. Early vertebral trabecular bone loss in normal premenopausal women. J. Bone Min. Res. 3:445-449, 1988.

7. Burger, E.H., and J. Klein-Nulend. Mechanotransduction in bone-role of the lacunocanalicular network. FASEB Journal, 13(suppl.): S101-S112, 1999.

8. Davies, K.M., R.R. Recker, M.R. Stegman, R.P. Heaney, D.B. Kimmel, and J. Leist. Third decade bone gain in women. In: Calcium Regulation and Bone Metabolism, D.V. Cohn, F.H. Glorieux, and T.J. Martin (Eds.). Amsterdam: Elsevier Science, 1990, pp. 497-550.

9. Döbeln, W. von. Anthropometric determination of fat-free body weight. Acta. Med. Scand. 165:37, 1959.

10. von Döbeln, W. Determination of body constituents. In: Occurrence, causes and prevention of overnutrition, G. Blix (Ed.) Uppsala: Almquist and Wiksells, 1964, pp. 103-107.

11. Falkner, F., and J.M. Tanner. Human Growth, Part 1, 2 and 3. New York: Plenum Press, 1978.

12. Frost, H.M. An approach to estimating bone and joint loads and muscle strength in living subjects and skeletal remains. Am. J. Human Biol. 11:437-455, 1999.

13. Geusens, P., F. Cantatore, J. Nijs, W. Proesmans, F. Emma, and J. Dequeker. Heterogeneity of growth of bone in children at the spine, radius and total skeleton. Growth Dev. Aging 55:249-256, 1991.

14. Gilsanz, V., D.T. Gibbons, T.F. Roe, and M. Carlson. Vertebral bone density in children: effect of puberty. Radiology. 166:847-850, 1988.

15. Glastre, C., P. Braillon, L. David, P. Cochat, P.J. Meunier, and P.D. Delmas. Measurement of bone mineral content of the lumbar spine by dual energy X-ray absorptiometry in normal children: correlations with growth parameters. J. Clin. Endocrin. Metab. 70:1330-1333, 1990.

16. Gleeson, P.B., E.J. Protas, A.D. LeBlanc, V.S. Schneider, and H.J. Evans. Effects of weight lifting on bone mineral density in premenopausal women. J. Bone Min. Res. 5:153-158, 1990.

17. Gordon, C.L., J.M. Halton, S.A. Atkinson, and C.E. Webber. The contributions of growth and puberty to peak bone mass. 55:257-262, 1991.

18. Greulich, P., and S.I. Pyle. Radiographic Atlas of Skeletal Development of the Hand and Wrist. Stanford, CA: Stanford University Press, 1950 (1st ed.), 1959 (2nd ed.).

19. Grimston, S.K., K. Morrison, J.A. Harder, and D.A. Hanley. Bone mineral density during puberty in Western Canadian children. Bone and Min. Res. 19:85-96, 1992.

20. Groothausen, J., H. Siemer, H.C.G. Kemper, J.W.R. Twisk, and D.C. Welten. Influence of peak strain on lumbar bone mineral density: an analysis physical activity in young males and females. Pediatr. Exerc. Sci. 9:159-173, 1997.

21. Kannus, P., H. Haapasalo, M. Sankelo, H. Sievanen, M. Pasanen, A. Heinonen, P. Oja, and I. Vuori. Effect of starting age of physical activity on bone mass in the dominant arm of tennis and squash players. Ann. Intern. Med. 123:27-31, 1995. 
22. Kemper, H.C.G. The Amsterdam Growth and Health Study, Health, Fitness and Lifestyle in Longitudinal Perspective; A Follow-up of Males and Females from 13 to 27 Years of Age (monograph No. 6). Champaign, IL: Human Kinetics, 1995.

23. Kemper, H.C.G. Exercise to Fight Osteoporosis. A Guide for the Health Care Professional. Zeist: Medical Forum International, 1998.

24. Kemper, H.C.G., and R.A. Binkhorst. Exercise and the physiological consequences of the ageing process. In: Ageing, Health and Competence, J.J.F. Schroots (Ed.). Amsterdam: Elsevier, 1993, pp. 109-126.

25. Kontulainen, S., P. Kannus, H. Haapsalo, A. Heinonen, H. Sievänen, P. Oja, and I. Vuori. Changes in bone mineral content with decreased training in competitive young adult tennis players and controls: a prospective 4-year follow-up. Med. Sci. Sports Exerc. 31:640-652, 1999.

26. Lanyon, L.E. Using Functional loading to influence bone mass and architecture: objectives, mechanism, and relationship with estrogen of the mechanically adaptive process in bone. Bone. 18:37S-43S, 1996

27. Mirwald, R.L., D.A. Bailey, H. McKay, and P.E. Crocker. Physical Activity and Bone Mineral Acquisition at the Lumbar Spine During the Adolescent Growth Spurt. Abstract from the First International Conference on Children's Bone Health, Maastricht, 1999, p. 57.

28. Margulies, J.Y., A. Simkin, I. Leichter, A. Bivas, R. Steinberg, M. Giladi, M. Stein, H. Kashtan, and C. Milgrom. Effect of intensive physical activity on the bone mineral density content in power limbs of young adults. J. Bone Joint Surgery. 68A:1090-1093, 1986.

29. Moen, S., C. Sanborn, S. Bonnick, H. Keizer, B. Gench, and N. DiMarco. Longitudinal lumbar bone mineral density changes in adolescent female runners. Med. Sci. Sports Exerc. 24:S122, 1992.

30. Morris, F.L., G.A. Naughton, J.L. Gibbs, J.S. Carlson, and J.D. Wark. Positive effects on bone and lean mass. J. Bone Min. Res. 12:1453-1462, 1997.

31. Recker, R.R., K.M. Davies, S.M. Hinders, R.P. Heaney, R.P. Stegman, and D.B. Kimmel. Bone gain in young adult women. JAMA. 268:2403-2408, 1992.

32. Rico, H., M. Revilla, E.R. Hernandez, L.F. Villa, and L. Alvarez del Buergo. Sex differences in the acquisition of total bone mineral mass peak assessed through dual energy X-ray absorptiometry. Calcif. Tissue Int. 51:251-254, 1992.

33. Riggs, B.L., and L.J. Melton. Involutional osteoporosis. N. Engl. J. Med. 413:16761686, 1986.

34. Riis, B.J., S. Krabbe, C. Christiansen, D.B. Catherwood, and L.J. Deftos. Bone turnover in male puberty: a longitudinal study. Calcif. Tis. Int. 37:213-217, 1985.

35. Roche, A.F., H. Wainer, and D. Thissen. Skeletal Maturity: The Knee Joint as a Biological Indicator. New York: Plenum, 1975.

36. Roche, A.F., W.C. Chumlea, and D. Thissen. Assessing the Skeletal Maturity of the Hand-Wrist: Fels Method. Springfield, IL: C.C. Thomas, 1988.

37. Rubin, C.T., and L.E. Lanyon. Regulation of bone formation by applied dynamic loads. J. Bone Joint Surg. 66A:397-402, 1984.

38. Sievänen, H., P. Kannus, V. Nieminen, A. Heinonen, P. Oja, and I. Vuori, I. Estimation of various mechanical characteristics of human bones using DEXA: methodology and precision. Bone. 18:173-275, 1966.

39. Slemenda, C.W., J.Z. Miller, L.S. Hui, T.K. Reister, and C.C. Johnston. Role of physical activity in the development of skeletal mass in children. J. Bone Min. Res. 6:12271233, 1991. 
40. Smith, E.L., and D.M. Raab. Osteoporosis and physical activity. Acta. Medica. Scand. 711(Suppl.):149-156, 1986.

41. Snow-Harter, C., and R. Marcus. Exercise, bone mineral density and osteoporosis. Exerc. Sport Sci. Rev. 19:351-388, 1991.

42. Southard, R.N., J.D. Morris, J.R. Hayes, M.A. Torch, and A. Sommer. Bone mass in healthy children: measurement with quantitative DXA. Radiology. 179:735-738, 1991.

43. Tanner, J.M. Growth at Adolescence. Oxford, UK: Blackwell, 1955.

44. Tanner, J.M. A History of the Study of Human Growth. London: Cambridge University Press, 1981.

45. Tanner, J.M., R.H. Whitehouse, W.A. Marshall, M.J.R. Healy, and H. Goldstein. Assessment of Skeletal Maturity and Prediction of Adult Height (TW2 Method). London: Academic Press, 1975.

46. Theintz, G., B. Buchs, R. Rizolli, D. Slosman, H. Clavien, P.C. Sizonenko, and J.P.H. Bonjour. Longitudinal monitoring of bone mass accumulation in healthy adolescents: evidence for a marked reduction after 16 years of age at the levels of lumbar spine and femoral neck in female subjects. J. Clin. Endocrin. and Metab. 75:1060-1066, 1992.

47. Weiner, J.S., and J.A. Lourie. Human Biology, a Guide to Field Methods IBP Handbook No. 9. Oxford, UK: Blackwell, 1969.

48. Welten, D.C., H.C.G. Kemper, G.B. Post, W.v. Mechelen, J. Twist, P. Lips, and G.J. Teule. Weight bearing activity during youth is a more important factor for peak bone mass than calcium intake. J. Bone Min Res. 9:1089-1096, 1994.

49. Wolff, I., I.I. Croonenberg, H.C.G. Kemper, P.J. Kostense, and J.W.R. Twisk. The effect of exercise training programs on the bone mass: a meta-analysis of published controlled trials in pre- and postmenopausal women. Osteopor. Int. 9:1-12, 1999. 